\title{
Comparison of Changes in the Interstitial Cells of Cajal and Neuronal Nitric Oxide Synthase-positive Neuronal Cells With Aging Between the Ascending and Descending Colon of F344 Rats
}

\author{
Sun Min Lee, ${ }^{1}$ Nayoung Kim, ${ }^{1,2 *}$ Hyun Jin Jo, ${ }^{1}$ Ji Hyun Park, ${ }^{2}$ Ryoung Hee Nam, ${ }^{1}$ Hye Seung Lee, ${ }^{3}$ Hyun Jin Kim, ${ }^{4}$ Moon Young Lee, ${ }^{5}$ \\ Yong Sung Kim, ${ }^{6}$ and Dong Ho Lee ${ }^{1}$ \\ ${ }^{I}$ Department of Internal Medicine, Seoul National University Bundang Hospital, Seongnam, Gyeonggi-do, Korea; ${ }^{2}$ Department of Internal Medicine \\ and Liver Research Institute, Seoul National University College of Medicine, Seoul, Korea; ${ }^{3}$ Department of Pathology, Seoul National University \\ Bundang Hospital, Seongnam, Gyeonggi-do, Korea; ${ }^{4}$ Department of Internal Medicine, Gyeongsang National University Changwon Hospital, \\ Gyeongsang National University College of Medicine, Jinju, Gyeongsangnam-do, Korea; ${ }^{5}$ Department of Physiology and Institute of Wonkwang \\ Medical Science, Wonkwang University College of Medicine, Iksan, Jeollabuk-do, Korea; ${ }^{6}$ Division of Gastroenterology and Wonkwang Digestive \\ Disease Research Institute, Department of Internal Medicine, Wonkwang University Sanbon Hospital, Gunpo, Gyeonggi-do, Korea
}

\section{Background/Aims}

Neuronal degeneration and changes in interstitial cells of Cajal (ICCS) are important mechanisms of age-related constipation. This study aims to compare the distribution of ICCS and neuronal nitric oxide synthase (nNOS) with regard to age-related changes between the ascending colon (AC) and descending colon (DC) in 6-, 31-, and 74-week old and 2-year old male Fischer-344 rats.

\section{Methods}

The amount of fecal pellet and the bead expulsion times were measured. Fat proportion in the muscle layer of the colon was analyzed by hematoxylin and eosin staining. Proto-oncogene receptor tyrosine kinase (KIT) and neuronal nitric oxide synthase (nNOS) expression were analyzed with Western blotting and immunohistochemistry. Isovolumetric contractile measurements and electrical field stimulation were used to assess smooth muscle contractility.

\section{Results}

Colon transit and bead expulsion slowed with senescence. Fat in the muscle layer accumulated with age in the AC, but not in the DC. The proportion of KIT-immunoreactive ICCs in the submucosal and myenteric plexus was higher in the DC than in the AC, and it declined with age, especially in the AC. In contrast, the proportion of NOS-immunoreactive neurons in the myenteric plexus was higher in the AC than in the DC, and both decreased in older rats. Nitric oxide levels declined with age in the DC. Muscle strip experiments showed that the inhibitory response mediated by nitric oxide in the circular direction of the DC was reduced in 2-year old rats.

\section{Conclusion}

The AC and DC differ in their distribution of ICCS and nNOS, and age-related loss of nitrergic neurons more severely affects the DC than the AC.

(J Neurogastroenterol Motil 2017;23:592-605)

Key Words

Aging; Colon; Interstitial cells of Cajal; Nitric oxide synthase type I; Rats, inbred F344

Received: April 30, 2017 Revised: June 20, 2017 Accepted: July 9, 2017

(a) This is an Open Access article distributed under the terms of the Creative Commons Attribution Non-Commercial License (http://creativecommons. org/licenses/by-nc/4.0) which permits unrestricted non-commercial use, distribution, and reproduction in any medium, provided the original work is properly cited.

${ }^{*}$ Correspondence: Nayoung Kim, MD Department of Internal Medicine, Seoul National University Bundang Hospital, 82 Gumi-ro 173beon-gil, Bundang-gu, Seongnam, Gyeonggi-do 13620, Korea

Tel: +82-31-787-7008, Fax: +82-31-787-4051, E-mail: nayoungkim49@empas.com 


\section{Introduction}

Constipation is one of the most common digestive diseases. The prevalence of constipation is $12-19 \%$, and it increases with age. ${ }^{1,2}$ Among constipation patients over 65 years old, $65 \%$ reported symptoms of straining during bowel movements and $40 \%$ reported symptoms of excessively solid stools. ${ }^{3}$ Furthermore, the prevalence of constipation was $26 \%$ in women and $16 \%$ in men 65 years of age or older in a study on self-reported constipation. ${ }^{4}$ Age-related changes in the lower gastrointestinal tract include delayed colon transit time and reduction of the moisture content of stools. ${ }^{5}$ These changes can result from intestinal atrophy, reduced blood supply, and alterations in the enteric nervous system. ${ }^{6}$

Interstitial cells of Cajal (ICCs) are the pacemaker cells in the colon; they induce spontaneous electrical and mechanical activity by acting as intermediaries of neurotransmission. ${ }^{7-9} \mathrm{~A}$ decline in the number and volume of ICCs with age was reported in a study in normal human colons. ${ }^{10}$ KIT protein, which is the receptor for stem cell factor, is expressed in ICC and is an established immunohistological marker for ICCs. ${ }^{7,11}$ Kit mRNA and KIT protein levels decline in the colons of patients with slow-transit constipation. ${ }^{12,13}$ In addition, stem cell factors and KIT protein activity is closely related to the development and sustenance of ICCs, and their expression levels decrease with age in the rat ascending colon (AC). ${ }^{14-16}$

Neuronal degeneration in the enteric nervous system is an important mechanism of age-related functional changes in the guts of older animals. ${ }^{17}$ Dysfunction of intestinal muscles is associated with a reduction in enteric neuronal cells and an impaired response to direct stimuli in the myenteric plexus. ${ }^{18,19}$ Similar findings have been demonstrated in aging mice, rats, guinea pigs, and a nonhuman primate model. ${ }^{20-23}$ The extent of age-related loss of enteric neurons is controversial to date. In humans and rats, selective loss of cholinergic neurons with sparing of nitrergic neurons has been reported. $^{24-26}$ In contrast, there was a report of a decrease of neuronal nitric oxide synthase (nNOS) in old rats. ${ }^{27}$ On the other hand, restricting diet prevented age-related myenteric neuronal loss in the ileum of Sprague-Dawley rats. ${ }^{28}$ Nitrergic neurons generate nitric oxide (NO) by nNOS to regulate the non-adrenergic non-cholinergic relaxation of smooth muscle that affects colonic motility. ${ }^{29} \mathrm{NO}$ enhances colonic transit by mediating descending relaxation and facilitates propulsion of the contents of the colon. ${ }^{30}$ Age-related loss of $\mathrm{nNOS}$ in rats was reported in the AC and mid-colon; ${ }^{14,27}$ however, it has not been studied in the descending colon (DC) of the rat. Although the extent of neuronal loss differed in human and rats, the region-specific neuronal change in DC remains to be investigated.

The colon has embryological and functional regional differences between the AC and DC. That is, the AC and DC come from different embryological origins and have distinct features in terms of development of ICCs at the end of the embryonic period in humans. ${ }^{31}$ Motor patterns also differ according to colonic regions. ${ }^{32}$ The velocity of fecal pellet propulsion is higher in the distal colon than in the mid- and proximal colons in guinea pigs. ${ }^{32}$ These studies imply that there may be some distinctive features of ICCs and NOS associated with the differences in the decline of colonic motility that happens with age between the AC and DC. Several studies have shown the distribution of ICCs and $\mathrm{nNOS}$ in the AC and DC; however, the results varied possibly because of differences in ages and species. ${ }^{33-37}$ Since there is loss or scathe of ICCs and neurons in the aging colon, we hypothesized that the distribution of ICCs and nNOS may change as animals age, and the functional consequences could be different according to the colonic site. Given this background, the aim of this study is to investigate the differential distribution of ICCs and nNOS with regard to age-related changes between the AC and DC in male Fischer-344 (F344) rats. In relation to these anatomical changes, functional studies were also performed in the 6-, 31-, and 74-week old and 2-year old rats.

\section{Materials and Methods}

\section{Animals}

Specific-pathogen-free, male F344 rats 6, 31, and 74 weeks, and 2 years of age were used in these studies (Orient Co Ltd, Seoul, Korea). The animals were bred under a specific-pathogen-free condition, at $23^{\circ} \mathrm{C}$ and $12: 12$-hour light-dark cycles. They were provided with unrestricted access to food and water. This study was performed in strict accordance with the recommendations in the Guide for the Care and Use of Laboratory Animals of South Korea. All experiments were approved by the Institutional Animal Care and Use Committee (IACUC) of Seoul National University Bundang Hospital (Permission No. BA1304-27/033-04).

\section{Assessment of Constipation-like Phenotype in Rats' Defecation}

Starting from 8-9 AM, the rats (in each age group, $\mathrm{n}=8$ ) were fasted for 24 hours with free access to tap water. They were then allowed access to food for 2 hours, and were fasted again for 24 hours in order to observe defecation. ${ }^{38}$ During fasting, we avoided the use of wire mesh in order to reduce stress. We recorded the 
weight of the provided food before and after feeding. After feeding, we recorded the number of stools produced over the course of 4 hours and 24 hours to investigate total fecal output including late defecation. To measure dry fecal weight, we collected the fecal samples that were excreted over the course of 24 hours and dried them for an additional 24 hours. The results are described as number of defecations per rat and dry fecal weight per $300 \mathrm{~g}$ body weight.

\section{Bead Expulsion Study}

Each experiment started between 8 and 9 AM. Before the experiment, rats (in each age group, $\mathrm{n}=8$ ) fasted for 24 hours but given access to water. The fasted animals were then fed for 2 hours. We anesthetized the animals with isoflurane, eliminated stools in the rectum and inserted colored plastic beads to a depth of $3 \mathrm{~cm}$ past the anus using a plastic rod. The diameter of the beads was $5 \mathrm{~mm}$ for 6-week old rats and $6 \mathrm{~mm}$ for rats of other ages. After placing each rat in an individual cage, we observed the rats for 4 hours to measure time until bead expulsion.

\section{Tissue Preparation}

After the defecation study and bead expulsion study, animals were terminally anesthetized via inhalation of carbon dioxide to obtain colonic tissue samples. After discarding $1 \mathrm{~cm}$ from the cecum and anus, $5 \mathrm{~cm}$ of the proximal and distal parts of the colon were collected from each. ${ }^{33,39}$ A part of each sample $1 \mathrm{~cm}$ in length was prepared for histological analysis by fixation in $10 \%$ buffered formalin. The tissue specimens embedded in paraffin blocks were cut perpendicularly to the lumen into $4 \mu \mathrm{m}$ thick sections and then stained with hematoxylin and eosin. Western blot samples were immediately frozen in liquid nitrogen, and stored at $-80^{\circ} \mathrm{C}$ until use.

\section{Measurement of Histologic Fat Proportion}

A pathologist blinded to the experimental groups analyzed the histology of the samples. One slide per animal (in each age group, $\mathrm{n}=10$ ) and 2 fields per slide were selected at random. The fat proportion in the muscle layer was estimated as the area of fatty tissue to total smooth muscle. The area was measured with the ImagePro Plus analysis system (Media Cybernetics, Inc., San Diego, CA, USA) and described as $\mathrm{mm}^{2}$ per field of view.

\section{Western Blotting for KIT and Neuronal Nitric Oxide Synthase}

We used AC and DC tissue after eliminating the mucosal layer. The colonic specimens were prepared for protein analysis, as previously described. ${ }^{14}$ Briefly, the samples (in each age group, $\mathrm{n}=6$ ) were homogenized in lysis buffer and the supernant was separated from the lysates. After SDS-PAGE, we transferred the proteins from gel to the PVDF membrane and blocked them with $5 \%$ skim milk. Starting with the blocking process, all procedures were performed with Tris buffer $(40 \mathrm{mM}, \mathrm{pH} 7.55)$ containing $0.3 \mathrm{M}$ of $\mathrm{NaCl}$ and $0.05 \%$ Tween 20. After blocking, we blotted the membranes with antibodies for KIT (1:100 dilution, rabbit polyclonal antibody; Santa Cruz Biotechnology, Santa Cruz, CA, USA), nNOS (1:500 dilution, mouse monoclonal IgG2a antibody; BD Biosciences, San Diego, CA, USA), and $\beta$-actin (1:1000 dilution, rabbit polyclonal antibody; Biovision, Milpitas, CA, USA) at $4^{\circ} \mathrm{C}$ overnight. Rabbit polyclonal antibodies (Santa Cruz Biotechnology) against KIT and $\beta$-actin and a mouse polyclonal antibody (Santa Cruz Biotechnology) against nNOS were used as secondary antibodies. For KIT data, the expression level was calculated by adding the band intensity of the mature and immature forms.

\section{Immunohistochemistry for KIT, Protein Gene Product 9.5, and Neuronal Nitric Oxide Synthase}

Immunohistochemistry (IHC) for KIT, protein gene product (PGP) 9.5, and nNOS was performed as previously described. ${ }^{14}$ The tissue sections were treated with $3 \%$ hydrogen peroxide and nonspecific binding sites were blocked. Then, the sections were incubated with primary antibodies. Anti-KIT (1:100 dilution, polyclonal rabbit anti-human CD117; DAKO, Glostrup, Denmark), anti-PGP 9.5 (1:250 dilution, CM 329 AK; Biocare Medical, Concord, CA, USA), and anti-nNOS (1:500 dilution, AB5380; EMD Millipore, Billerica, MA, USA) were used as the primary antibodies. Since mast cells are known to express KIT, they were excluded from the counts after being identified by their round or oval shape and deficiency of processes. ${ }^{7,10,40}$ For immunostaining, an automatic immunostainer (BenchMark XT; Ventana Medical Systems, Inc, Tucson, AZ, USA) and UltraView Universial DAB detection kit (Ventana Medical Systems) were used. Sections not treated with primary antibodies were used as negative controls.

\section{Measurement of Nitric Oxide Levels}

NO was quantified using a microplate assay (G-7921, Thermo Fisher Scientific) following the manufacturer's protocol. Briefly, the colonic samples (in each age group, $n=4$ or $n=5$ ) were mixed with a reagent containing equal volumes of $\mathrm{N}$-(1-naphthyl)ethylenediamine and sulfanilic acid, and then incubated for 30 minutes at room temperature. The absorbance of the nitrite-containing samples was measured in a spectrophotometric microplate reader. Measurements were taken at a wavelength of $548 \mathrm{~nm}$. 


\section{Assessment of Smooth Muscle Contractility}

Change in smooth muscle contractility was evaluated by measuring the contractile response with or without electrical field stimulation (EFS) and neuronal inhibitors. We used standard organ-bath techniques and methods for isovolumetric contractile measurement and EFS, as previously described. ${ }^{14}$ Five animals were used in each age group. The muscle strips were allowed to equilibrate for up to 1-2 hours before the experiments. Contractile activity was quantified by calculating the area under the curve (AUC). It was first measured in the resting state and then measured with EFS. EFS (320 $\mathrm{mA}$ and 1 millisecond pulse-width for 5 seconds) was performed at various frequencies of $1,2,5,10$, and $20 \mathrm{~Hz}$. The contractile response to potassium chloride $(\mathrm{KCl})$ was quantified to investigate non-neuronally mediated smooth muscle contractility. Contraction and relaxation of the colonic muscle induced by EFS was sequentially evaluated under atropine $(10 \mu \mathrm{M})$. Moreover, we assessed the EFS responses to apamin $(1 \mu \mathrm{M})$ and L-NG-nitroarginine methyl ester (L-NAME, $100 \mu \mathrm{M})$ under non-adrenergic non-cholinergic conditions by adding atropine, propranolol, and phentolamine (1 $\mu \mathrm{M}$ each). All data were obtained using the PowerLab data acquisition system, and the AUC was calculated using the values acquired immediately before and after EFS. The value obtained before drug treatment was defined as $100 \%$ when calculating the percent changes of EFS responses.

\section{Statistical Methods}

All statistical analyses were performed using the SPSS version 18 (IBM Corp, Armonk, NY, USA). For comparison of two means, Student's $t$ test or the Mann-Whitney test was used after normality evaluation with the Kolmogorov-Smirnov test. The correlation between 2 factors was determined with Pearson's simple correlation coefficient. Values are given as mean \pm SEM. A $P$-value of $<0.05$ was taken to indicate statistically significance.

\section{Results}

\section{The Effects of Aging on the Defecation}

The number of stools produced in 4 hours was less in 31-week old, 74-week old, and 2-year old rats than in 6-week old rats $(P=$ $0.009,31$-week old rats vs 6 -week old rats; $P<0.001,74$-week old rats vs 6 -week old rats; and $P<0.001,2$-year old rats vs 6-week old rats) (Fig. 1A). Total fecal number in 24 hours also diminished in older rats as compared to 6-week old and 31-week old rats $(P=$
0.007, 31-week old rats vs 6-week old rats; $P=0.002,74$-week old rats vs 6-week old rats; $P<0.001,2$-year old rats vs 6-week old rats; and $P=0.003,31$-week old rats vs 2 -year old rats) (Fig. 1A). Then the weight of the total feces produced over the course of 24 hours was determined and adjusted to body weight. The fecal weight declined in older rats; that is, it was less in 31-week old, 74 week old, and 2-year old rats than in 6-week old rats, and was also less in 2-year old rats as compared to 31 -week old rats $(P<0.001$, 31 -week old rats vs 6-week old rats; $P<0.001$, 74-week old rats vs 6-week old rats; $P<0.001,2$-year old rats vs 6-week old rats; and $P=0.049$, 2-year old rats vs 31-week old rats) (Fig. 1B). There was no correlation between the amount of food intake and fecal weight, as determined with Pearson's simple correlation coefficient $(P=0.073)$.

\section{The Effect of Aging on Distal Colonic Transit}

Following the evaluation of dysmotility in the whole colon, we investigated dysmotility specifically in the DC and rectum by measuring the time necessary for bead expulsion. The colonic bead expulsion time increased in 2-year old rats as compared to 6-week old rats $(P=0.043)$ (Fig. 1C), which indicates attenuation of distal colonic transit in older rats.

\section{Fat Proportion in the Ascending Colon and Descending Colon}

We analyzed fat deposition of the muscle layer on histology and compared the fat proportion between the AC and DC. Similar to what was found in a previous report, ${ }^{14}$ the proportion of fat in $74-$ week old rats and 2-year old rats was significantly higher in the $\mathrm{AC}$ than in $\mathrm{DC}$ ( $P=0.031$ and $P=0.004$, respectively) (Fig. 2$)$. On the other hand, the proportion of fat in the DC was extremely low; it was $0.00 \%$ in 74 -week old and $0.81 \%$ in 2-year old rats (Fig. 2).

\section{Comparison of KIT Expression Between the Ascending Colon and Descending Colon and the Effect of Aging: Western Blot}

KIT expression was higher in the DC than in the $\mathrm{AC}$, and there were statistically significant differences between 6-, 31-, and 74-week old rats $(P=0.013$ in 6-week old rats; $P<0.001$ in 31 week old rats; and $P=0.008$ in 74-week old rats) (Fig. 3A). The KIT protein level decreased in older ages; in the AC, it was lower in 31-week old, 74-week old, and 2-year old rats than in 6-week old rats $(P=0.034,31$-week old rats vs 6 -week old rats; $P=0.048$, 74-week old rats vs 6-week old rats; and $P=0.002$, 2-year old rats vs 6-week old rats) (Fig. 3A). Similarly, the KIT protein level in the 

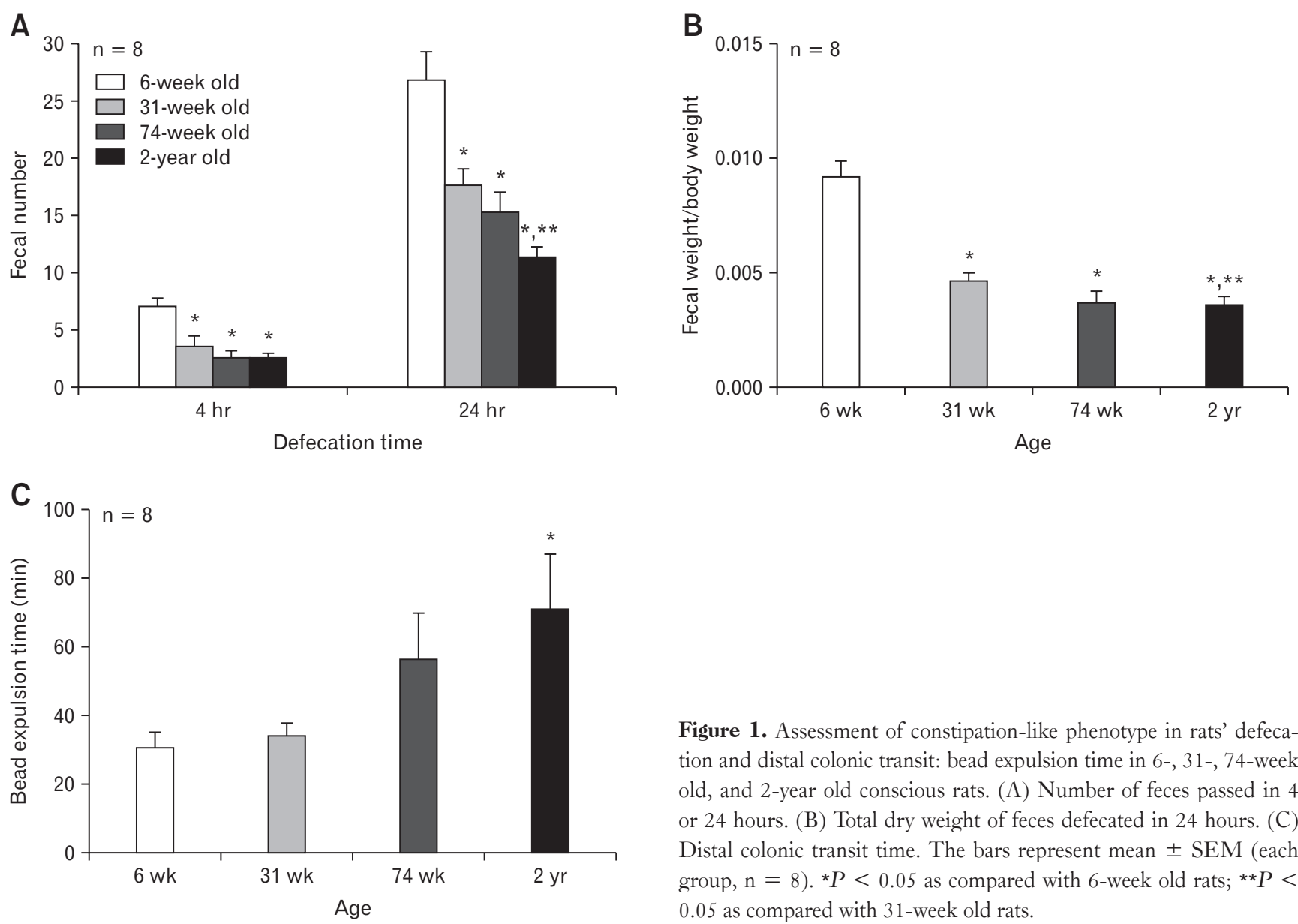

Figure 1. Assessment of constipation-like phenotype in rats' defecation and distal colonic transit: bead expulsion time in 6-, 31-, 74-week old, and 2-year old conscious rats. (A) Number of feces passed in 4 or 24 hours. (B) Total dry weight of feces defecated in 24 hours. (C) Distal colonic transit time. The bars represent mean \pm SEM (each group, $\mathrm{n}=8)$. ${ }^{*} P<0.05$ as compared with 6 -week old rats; ${ }^{*} P<$ 0.05 as compared with 31 -week old rats.

A

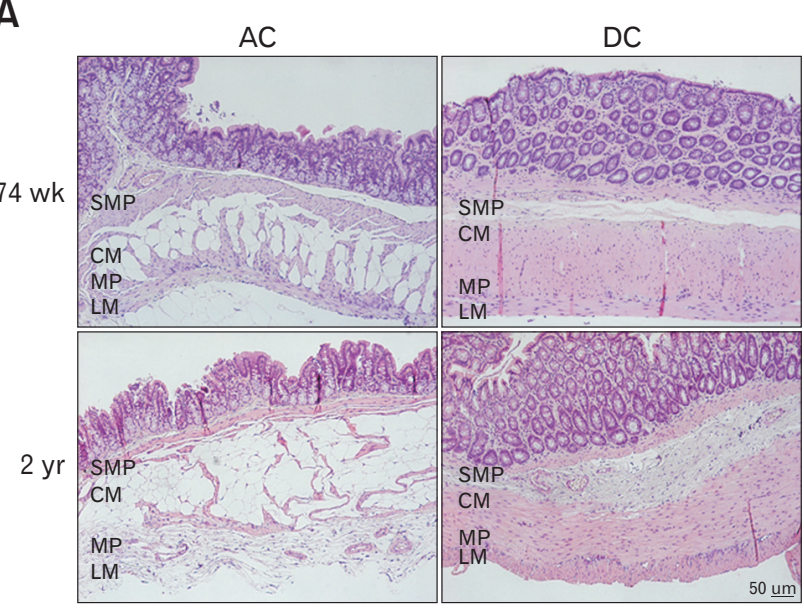

B

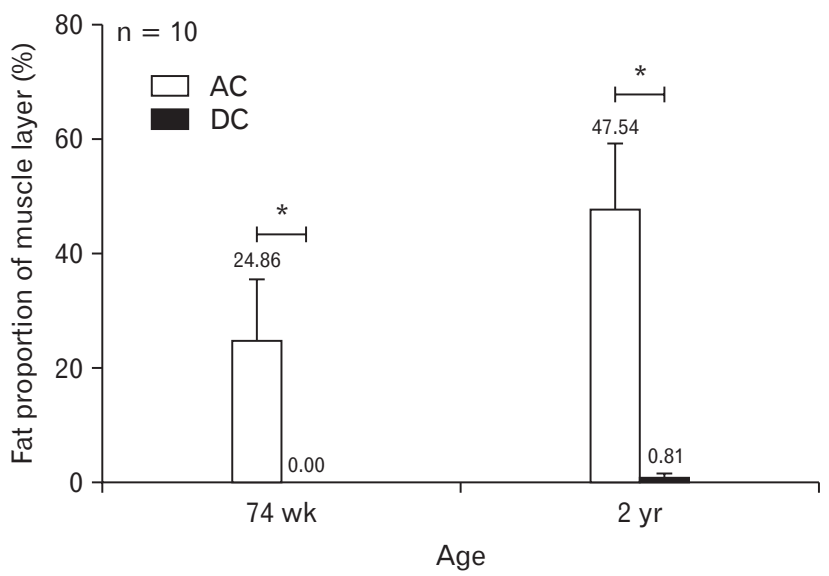

Figure 2. Proportion of fat in the muscle layer (\%). Fat proportion was investigated with histological analysis. (A) Hematoxylin and eosin stain $(\times 200$ magnifications) and (B) analysis of each measured area (mean \pm SEM). The mean values are labeled on each column $($ each group, $\mathrm{n}=$ 10). ${ }^{*} P<0.05$ between the ascending colon $(\mathrm{AC})$ and descending colon (DC). SMP, submucosal plexus; CM, circular muscle; MP, myenteric plexus; LM, longitudinal muscle. 
A
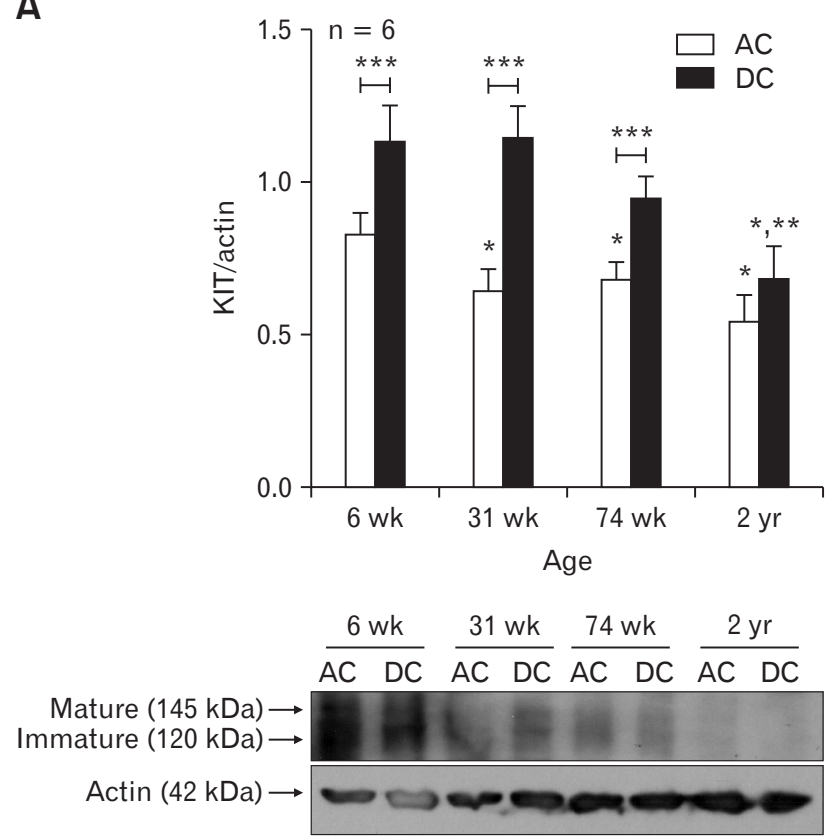

B
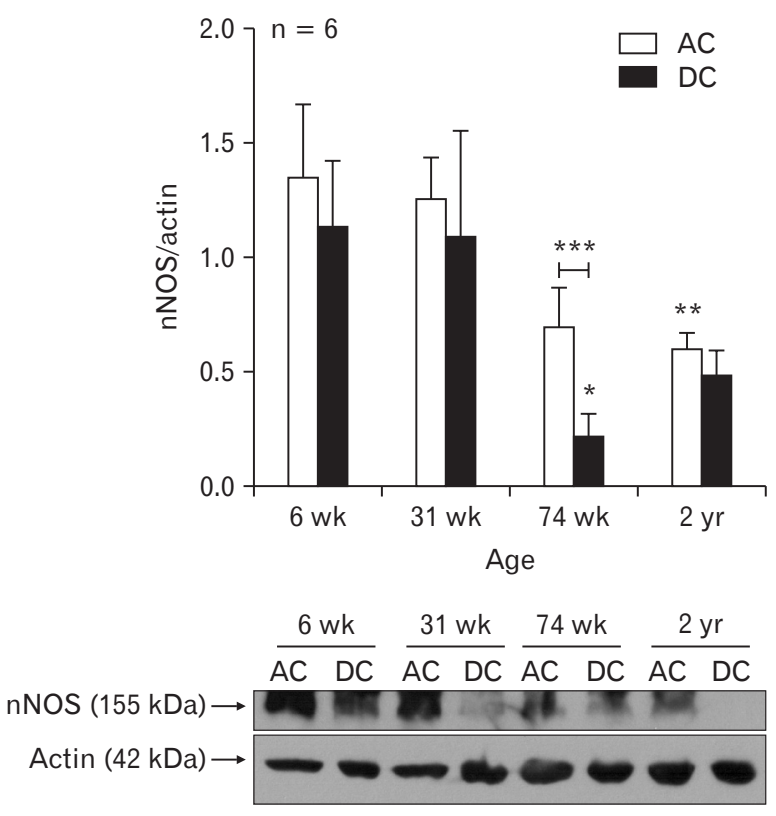

Figure 3. Expression of KIT protein and neuronal nitric oxide synthase (nNOS) in the rat ascending colon (AC) and descending colon (DC): changes by age and difference according to colonic region. (A) KIT protein level. (B) nNOS protein level. The bars represent the mean \pm SEM (each group, $\mathrm{n}=6$ ). ${ }^{*} P<0.05$ as compared with 6 -week old rats; ${ }^{* *} P<0.05$ as compared with 31 -week old rats; ${ }^{* *} P<0.05$ between the AC and DC.

DC was lower in 2-year old rats than in 6-week old and 31-week old rats $(P=0.018,2$-year old rats vs 6-week old rats; $P=0.011$, 2-year old rats vs 31-week old rats).

\section{Comparison of Neuronal Nitric Oxide Synthase Expression Between the Ascending Colon and Descending Colon and the Effect of Aging: Western Blot}

Comparing between the $\mathrm{AC}$ and $\mathrm{DC}$, in 74-week old rats, $\mathrm{nNOS}$ protein expression level was significantly higher in the $\mathrm{AC}$ than in the DC $(P=0.043)$ (Fig. 3B). The nNOS protein levels in the AC was significantly lower in 2-year old rats as compared to 31 -week old rats $(P<0.001)$. The nNOS expression in the DC significantly decreased in 74-week old rats as compared to 6-week old rats $(P=0.014)$; it also diminished in 2-year old rats but it was not statistically significant $(P=0.063)$ (Fig. 3B).

\section{Comparison of KIT-positive Area Between the Ascending Colon and Descending Colon and the Effect of Aging: Immunohistochemistry}

The KIT-positive area evident on IHC was higher in the DC than in the $\mathrm{AC}$ in rats of all 4 ages. However, there was a significant difference between the $\mathrm{AC}$ and $\mathrm{DC}$ in 74-week old and 2-year old rats in the submucosal plexus ( $P<0.001$ in both cases), and in 31 week old and 2-year old rats in the myenteric plexus $(P=0.041$ in 31 -week old rats and $P=0.001$ in 2-year old rats) (Fig. $4 \mathrm{~A}$ and $5 \mathrm{~A})$. This statistical difference of KIT expression levels between the $\mathrm{AC}$ and $\mathrm{DC}$ in relation to age originated from slightly different decreasing trends of KIT expression. That is, in the submucosal plexus, the KIT-positive area decreased with age specifically in the $\mathrm{AC}$ rather than in the DC (Fig. $4 \mathrm{~A}$ and $5 \mathrm{~A}$ ). In the myenteric plexus, the KIT-immunoreactive area was reduced in both the AC and DC.

\section{Comparison of Neuronal Nitric Oxide Synthase- positive Area Between the Ascending Colon and Descending Colon and the Effect of Aging: Immunohistochemistry}

There were differences in the nNOS-immunopositive area between the $\mathrm{AC}$ and $\mathrm{DC}$, such that the proportion of $\mathrm{nNOS}$-positive neurons was higher in the $\mathrm{AC}$ than in the $\mathrm{DC}$ in the myenteric plexus in 31-week old and 74-week old rats, but this difference was not found in the submucosal plexus (Fig. 4B and 5B). A decrease in the proportion of nNOS-positive neuronal cells in the DC was 
A

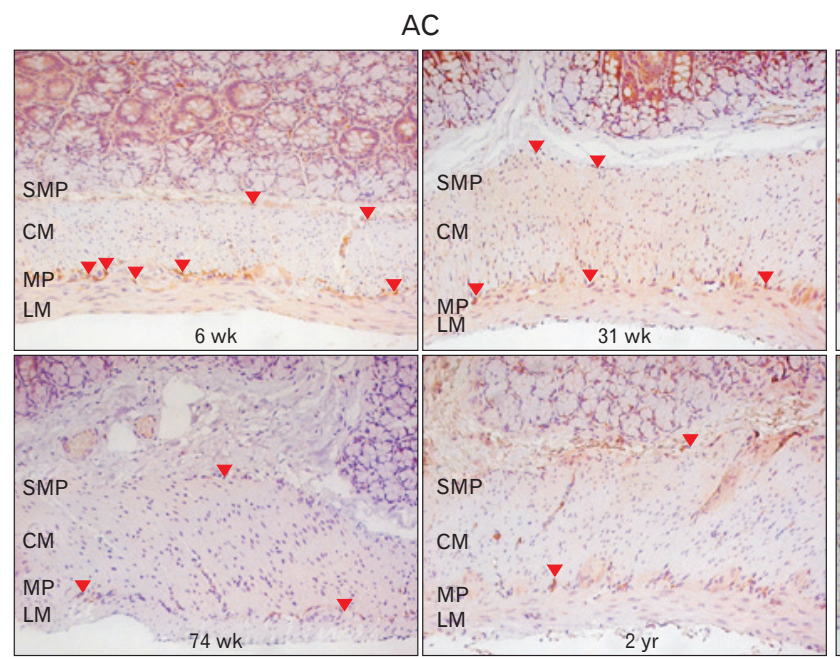

B

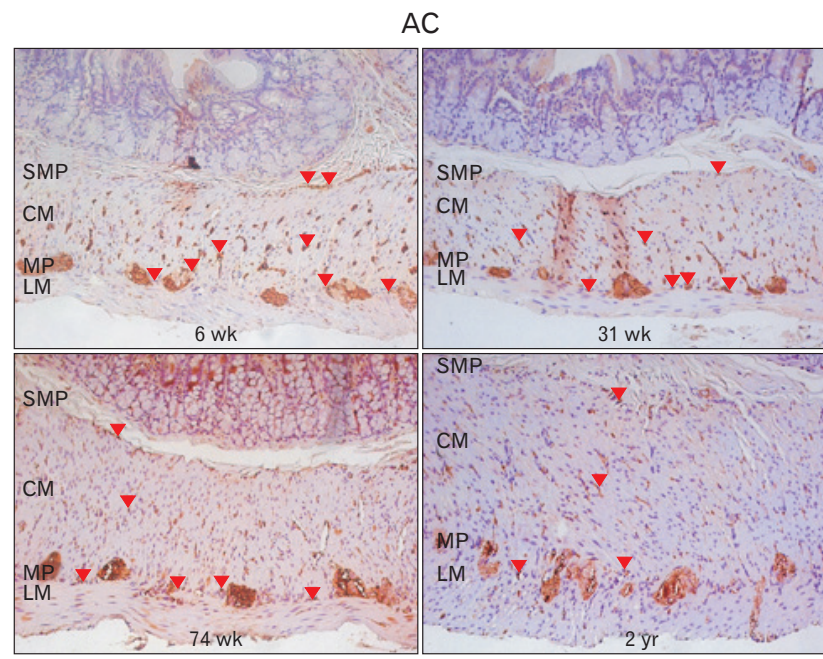

C

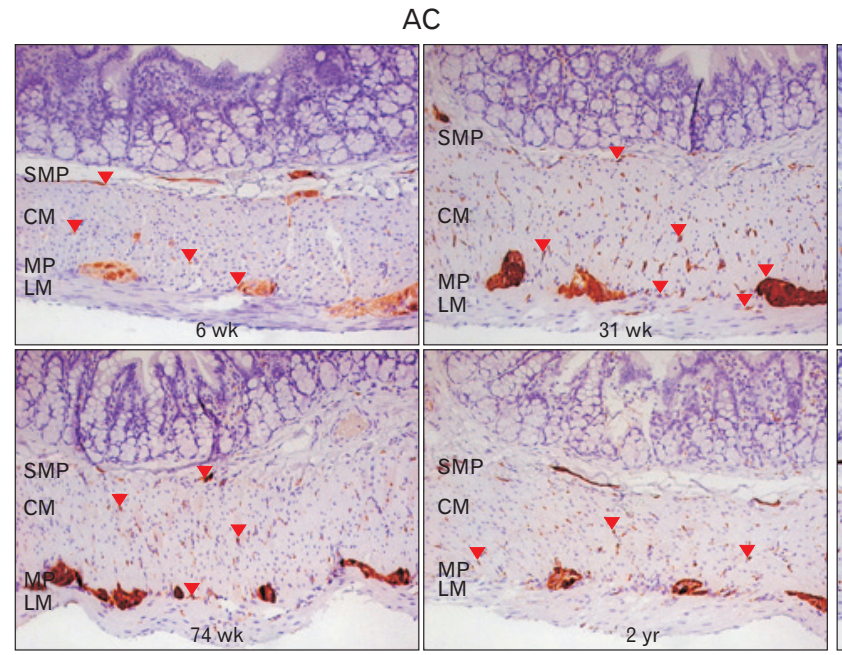

DC

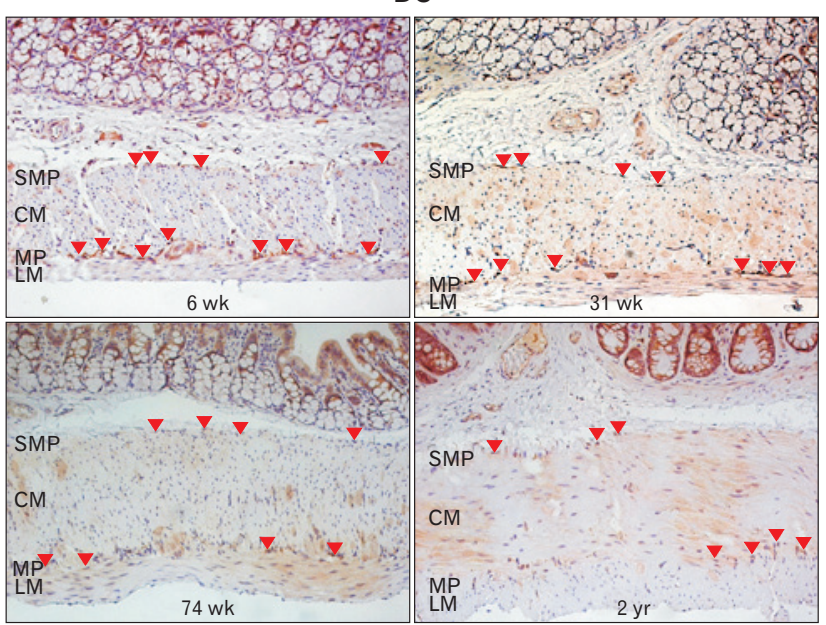

DC

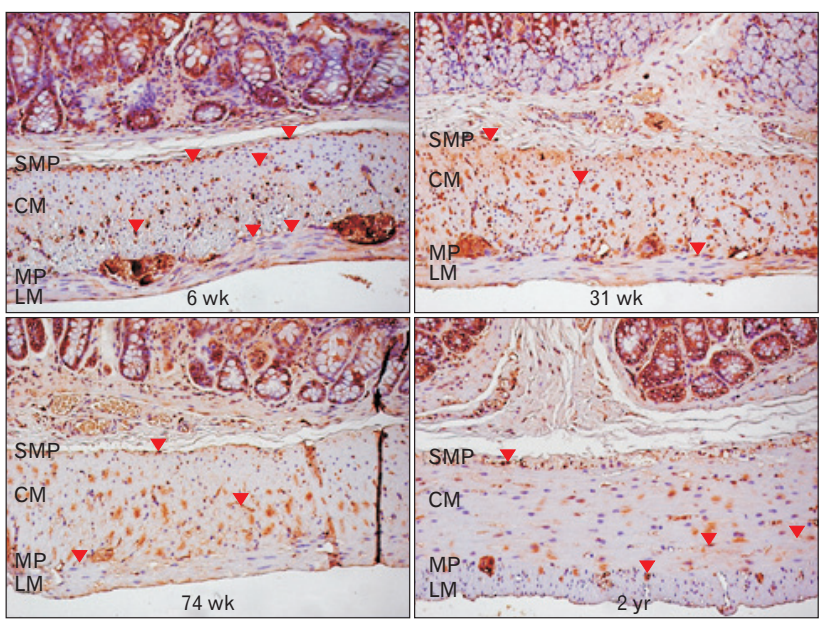

DC

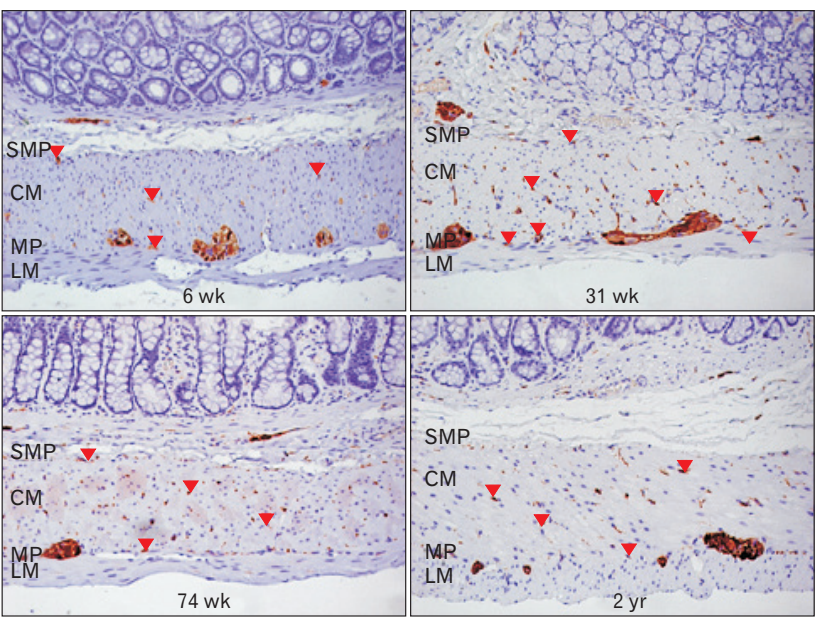

Figure 4. Photomicrography of KIT immunohistochemistry, neuronal nitric oxide synthase (nNOS), and protein gene product (PGP) 9.5 in the rat colon: changes according to age and difference according to colonic region. Arrowheads indicate (A) KIT-, (B) nNOS-, and (C) PGP 9.5-immunoreactive cells ( $\times 200$ magnification) in the ascending colon (AC) and descending colon (DC) of 6-, 31-, 74-week old, and 2-year old rats. SMP, submucosal plexus; CM, circular muscle; MP, myenteric plexus; LM, longitudinal muscle. 
A

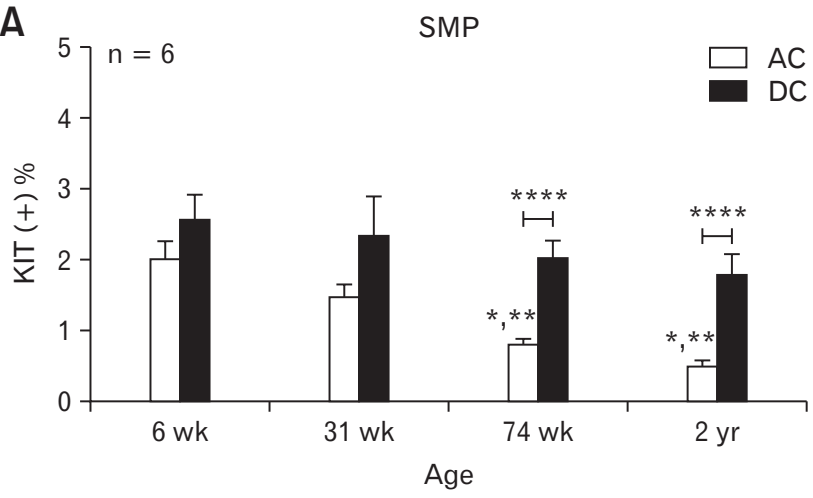

B
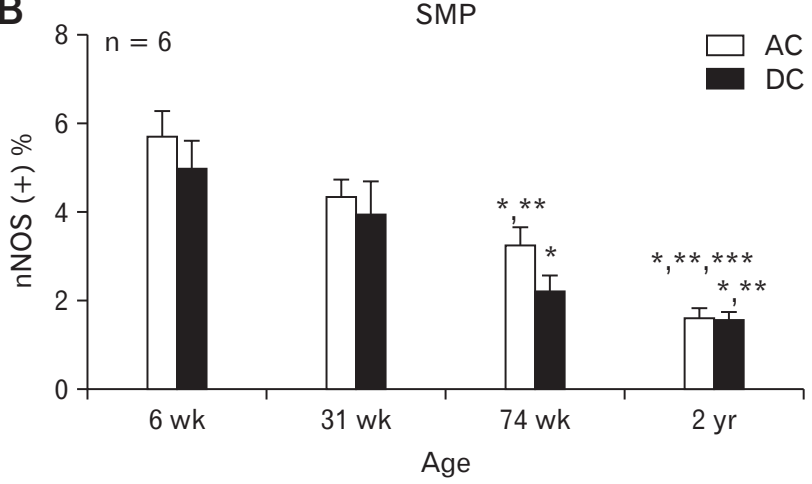

C
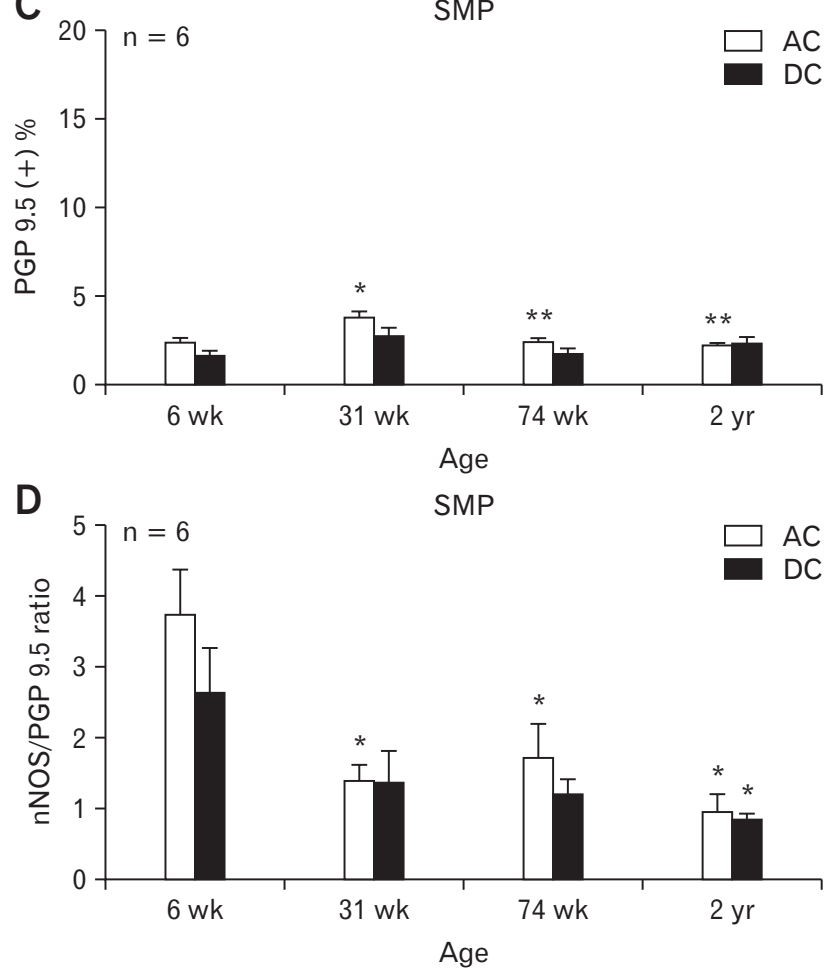
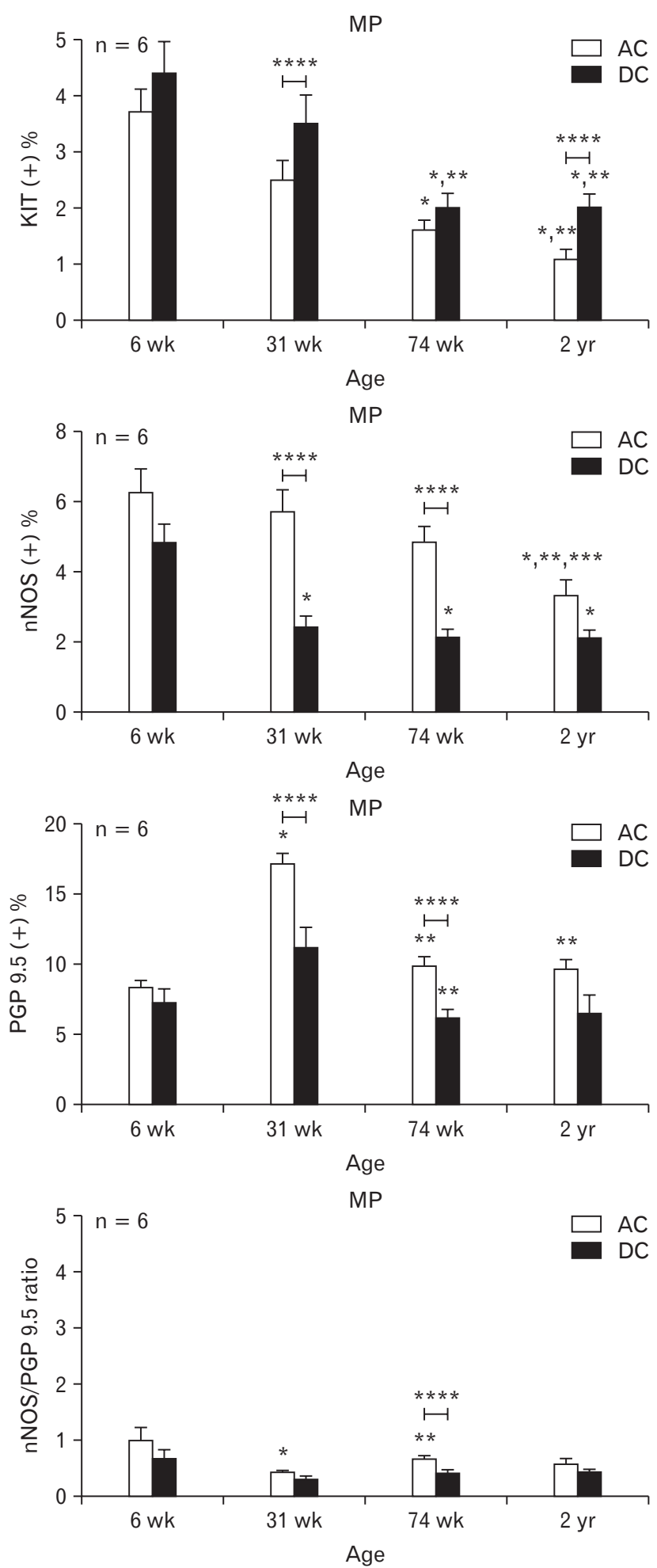

Fiğure 5. Immunohistochemical analysis of KIT, neuronal nitric oxide synthase (nNOS), and protein gene product (PGP) 9.5 in the rat colon: changes according to age and differences according to colonic region. The proportion of (A) KIT-, (B) nNOS-, (C) PGP 9.5-immunoreactive area, and (D) the nNOS/PGP 9.5 ratio in the submucosal plexus and myenteric plexus. The bars represents the mean \pm SEM (each group, $\mathrm{n}=$ 6). ${ }^{*} P<0.05$ compared to 6-week old rats; ${ }^{*} P<0.05$ compared to 31 -week old rats; ${ }^{* *} P<0.05$ compared to 74 -week old rats; ${ }^{* * *} P<0.05$ compared to ascending colon (AC). DC, descending colon; SMP, submucosal plexus; MP, myenteric plexus. 
detected beginning at the age of 31 weeks, which was earlier than in the AC, especially in the myenteric plexus. In detail, in the myenteric plexus, the proportion of nNOS-immunopositive area was lower in 31-week old and 74-week old rats than in 6-week old rats ( $P<0.001$ for all comparisons) (Fig. 4B and 5B). In the submucosal plexus of the DC, the proportion of the nNOS-positive area was lower in 74-week old rats than in 6-week old rats and in 2-year old rats, as compared to 6-week old rats and 31-week old rats $(P=$ 0.001, 74-week old rats vs 6-week old rats; $P<0.001$, 2 -year old rats vs 6 -week old rats; and $P=0.009,2$-year old rats vs 31 -week old rats) (Fig. 4B and 5B).

\section{Comparison of the Protein Gene Product}

9.5-positive Neuronal Structure Between the Ascending Colon and Descending Colon and the Effect of Aging: Immunohistochemistry

The proportion of PGP 9.5 immunopositive area was higher in the myenteric plexus than in the submucosal plexus (Fig. $4 \mathrm{C}$ and
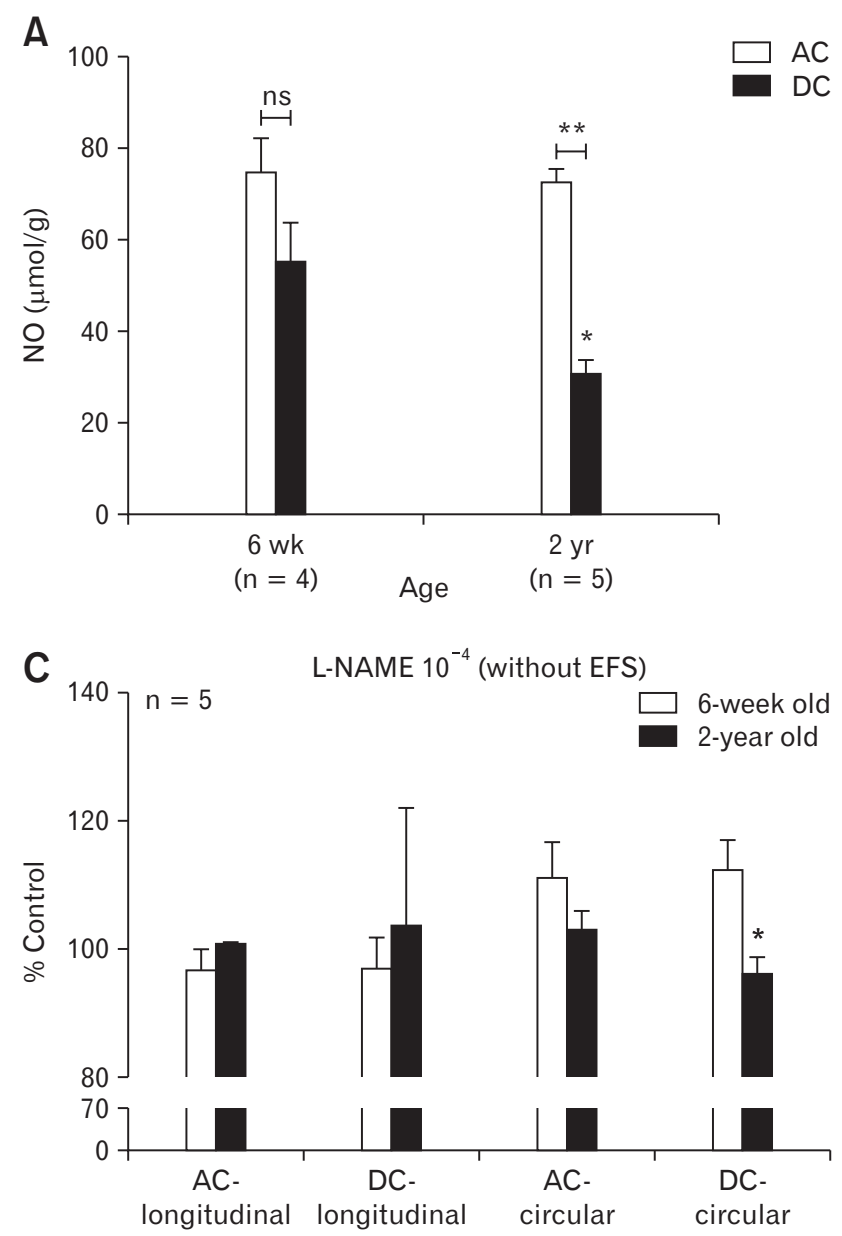

5C). In addition, in the myenteric plexus of 31-week old and 74week old rats, the proportion of PGP 9.5 immunoreactive area was significantly higher in the AC than in the DC $(P=0.003$ in 31 week old rats and $P=0.011$ in 74-week old rats) (Fig. 4C and $5 \mathrm{C})$, which is similar to the pattern of nNOS-positivity.

\section{Comparison of Neuronal Nitric Oxide Synthase/ Protein Gene Product 9.5 Between the Ascending Colon and Descending Colon and the Effect of Aging: Immunohistochemistry}

The nNOS/PGP 9.5 ratio was calculated as the percent of nNOS-positive area divided by the percent of PGP 9.5-positive area for each animal. In this analysis, the change of nNOS/PGP 9.5 ratio according to age was similar between the $\mathrm{AC}$ and DC (Fig. 5D). A decrease with age was more prominent in the submucosal plexus than in the myenteric plexus. In the submucosal plexus of the $\mathrm{AC}$, the ratio was lower in 31-week, 74-week and 2-year old rats as compared to 6 -week old rats $(P=0.012,31$-week old rats

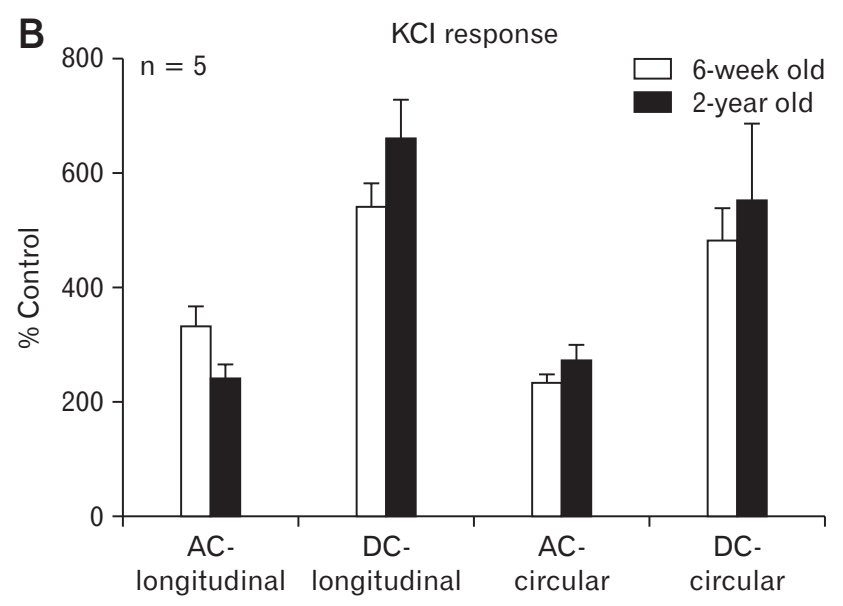

Figure 6. Nitric oxide (NO) levels and spontaneous contraction in 6-week old and 2-year old rat colons. (A) Changes in NO level according to age in the ascending colon (AC) and descending colon (DC). (B) Contractile responses to potassium chloride $(\mathrm{KCl})$ in the longitudinal or circular muscle in the AC and DC of 6-week old and 2-year old rats. (C) Spontaneous contraction in 6-week old and 2-year old rat colons after treatment with L-NG-nitroarginine methyl ester (L-NAME). Area under the curve of baseline contractile response during the contractile measurement. The bars represent the mean \pm SEM. The values shown are the differences in contraction before and after treatment with L-NAME at the resting state without electrical field stimulation (EFS). ${ }^{*} P<0.05$ as compared with 6-week old rat colon; ${ }^{* *} P<0.05$ between the AC and DC. 
vs 6 -week old rats; $P=0.028,74$-week old rats vs 6 -week old rats; and $P=0.005,2$-year old rats vs 6 -week old rats). It also decreased in the submucosal plexus of the DC in 2-year old rats as compared to 6-week old rats $(P=0.039)$. In the myenteric plexus of the $\mathrm{AC}$, the ratio decreased in 31 -week old rats $(P=0.036)$ and then increased in 74-week old rats $(P=0.013)$. There was no statistically significant change according to age in the myenteric plexus of DC.

\section{Nitric Oxide Levels}

$\mathrm{NO}$ has been suggested as an important factor for regulation of descending relaxation in the rat colon. ${ }^{41,42}$ The NO level was significantly decreased in 2-year old rats as compared to 6-week old rats in the $\mathrm{DC}(P=0.001)$, but not in the $\mathrm{AC}(P=0.791)$ (Fig. 6A). In addition, the discrepancy between the $\mathrm{AC}$ and $\mathrm{DC}$ was statisti-
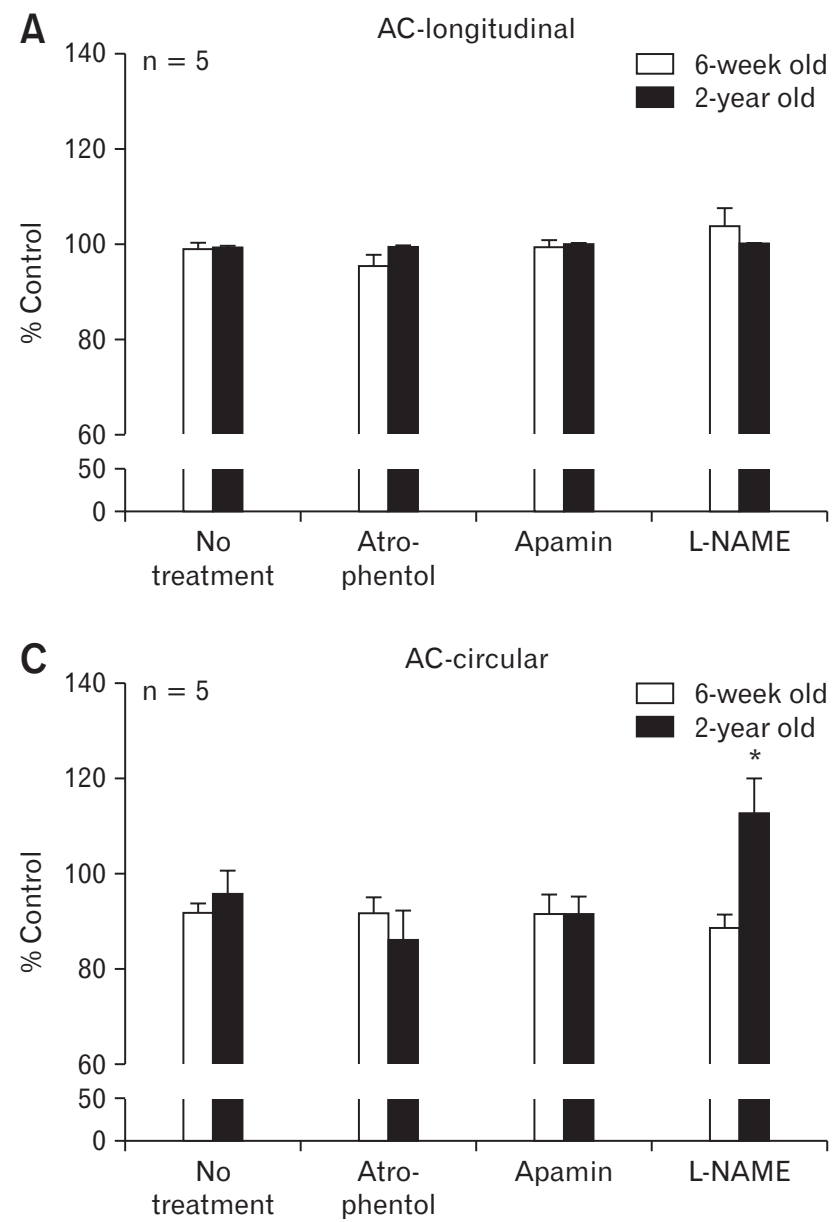

cally significant only in older animals $(P<0.001)$, not in younger animals $(P=0.174)$.

\section{Isovolumetric Contractile Measurement and Electrical Field Stimulation}

The response to $\mathrm{KCl}$ of 2-year old rats was analogous to that of 6-week old rats (Fig. 6B). The degree of spontaneous contraction in the circular direction of 6 -week old rats was over $100 \%$, which means that it increased after treatment with L-NAME (Fig. 6C). On the other hand, in 2-year old rats, especially in the circular direction in the DC, the contraction showed little discrepancy before and after treatment with L-NAME (Fig. 6C). The contractile response was lower in old rats than in young rats $(P=0.017)$ (Fig 6C).
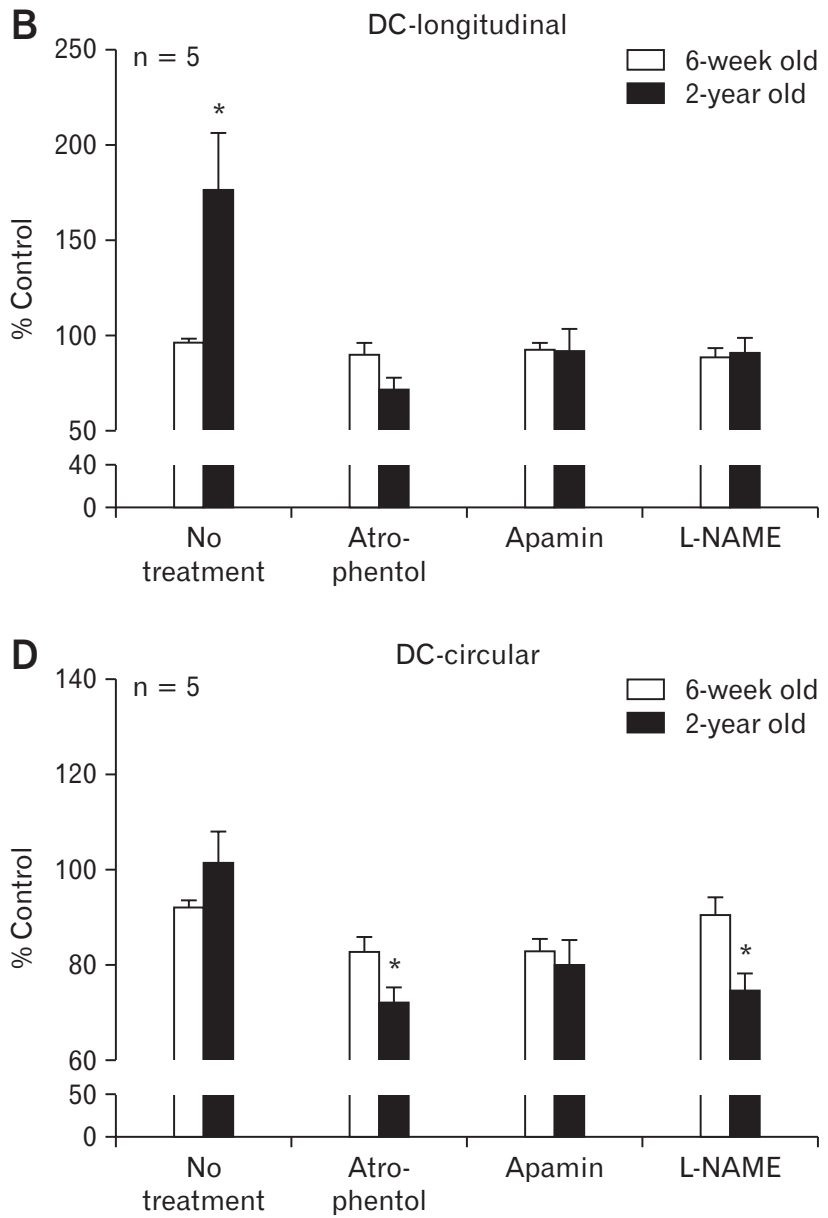

Figure 7. Electrical field stimulation (EFS) and contractile measurement with the colonic muscle strip in the ascending colon (AC) and descending colon (DC). EFS responses in the longitudinal direction in the AC (A) and DC (B), and in the circular direction in the AC (C) and DC (D). The response to EFS with atropine-phentolamine, apamin, and L-NG-nitroarginine methyl ester (L-NAME) were measured in the colons of 6-week old and 2-year old rats. The value before drug treatment was defined as $100 \%$ in the calculation of percent changes of EFS responses. All values are represented as mean $\pm \operatorname{SEM}$ (each group, $\mathrm{n}=5$ ). ${ }^{*} P<0.05$ as compared to 6 -week old rats. 
After electrical stimulation at $2 \mathrm{~Hz}$ for 5 seconds without any drug treatment, the contractile response significantly increased in 2 -year old rats in the distal longitudinal function $(P=0.033)$ (Fig. $7 \mathrm{~B})$. In the distal circular function, the EFS response significantly decreased in older rats, with treatment of atropine-phentolamine and L-NAME $(P=0.028$ with atropine-phentolamine and $P=$ 0.011 with L-NAME) (Fig. 7D). Degree of contraction barely increased after treatment with L-NAME, as compared to after treatment with apamin (Fig. 7D). The increase of longitudinal function in DC was also observed in every frequency (Supplementary Figure). The tendency was similar but there was no significant difference between the 6-week old rats and 2-year old rats in the EFS response at 1,5,10, and $20 \mathrm{~Hz}$, except for the decrease of EFS response with treatment of atropine-phentolamine in 2-year old rats stimulated with EFS at $20 \mathrm{~Hz}$ (Supplementary Figure P).

\section{Discussion}

The aim of this study was to compare the AC and DC of rats regarding the distribution and age-associated changes of ICCs and nNOS. Since we have reported age-related changes in the $\mathrm{AC}$ of Fisher rats in a previous study, ${ }^{14}$ the present study focused on investigating the differences of $\mathrm{AC}$ and DC. The results of our study demonstrate the following differences between the AC and DC: (1) fat deposition rarely occurred in the DC; (2) the expression of KIT in the submucosal plexus was higher in the DC than the AC and the expression of nNOS in the myenteric plexus was higher in the $\mathrm{AC}$ than the DC, and both decreased with age; and (3) the reduction of $\mathrm{NO}$ and its functional effect was more severe in the DC.

A reduction in colonic motility was confirmed by the experiments examining the amount that the rats defecated. Number of stools produced over 4 hours and 24 hours and fecal weight had similar results, revealing a constipation-like phenotype in older rats. Next, the bead expulsion test, an in vivo experiment, was used to estimate propulsion in the DC. ${ }^{43,44}$ An increase in the time necessary for bead expulsion with age indicated a decrease in colonic transit in the DC (Fig. 1C).

The present study demonstrated the fat proportion using histology in the DC. A previous report on fat deposition in the AC of old F344 rats suggested that fat accumulation is one of the changes that result in the colonic dysmotility seen in old age. ${ }^{14}$ In contrast to the $\mathrm{AC}$, fat deposition markedly decreased in the DC of 74-week old and 2-year old rats. Therefore, fat deposition may not be the cause of colonic dysmotility in the DC.

Regional differences of ICCs and nNOS in the colon are not completely understood to date. From the postnatal to adult periods, ICCs emerge and develop in a proximal-to-distal manner in human colons. ${ }^{31}$ Ward et $\mathrm{al}^{34}$ suggested that the density of ICCs in all histologic layers was highest in the $\mathrm{AC}$ and dissipated in the proximal-todistal direction in 20-30 day old C57BL/6 mice, according to IHC data acquired with a confocal microscope. ${ }^{34}$ In the normal human colon and rectum, the transverse colon has the highest density of ICCs amongst all colonic regions, as analyzed with IHC. ${ }^{35}$ Albertí et $\mathrm{al}^{33}$ suggested that there were no major differences in the density of KIT-positive ICCs between the AC, mid-colon, and DC in rats. However, there have been reports of normal human colons that reveal a larger population of KIT-positive cells in the DC than in the AC. ${ }^{36,37}$ The present study showed that there are more KIT-positive ICCs in the submucosal plexus of F344 rats in the DC than in the AC. These discrepancies in data may have resulted from differences in ages and species of the animals used. In the study that performed IHC in 8-10 weeks old Sprague-Dawley rats, nNOS expression was greatest in the mid-colon. ${ }^{33}$ However, nNOS-positive cells were not observed in the submucosal plexus, and the distribution of nNOS on whole mount showed no significant difference between the AC and DC. ${ }^{33}$ On the other hand, in the present study, the distribution of nNOS in the submucosal plexus was observed not to differ between the AC and DC. According to a comparison of nNOS expression in the myenteric plexus between the AC and DC, the distribution of nNOS was not significantly different at 6-weeks of age, which was similar to the age examined in the previous study. It seems notable that there was a significant difference in distribution at ages of 31 weeks and above (Fig. 5B).

It is controversial whether age-related dysmotility is due to a decrease in the amount of smooth muscle cells themselves or to damage to intermediate cells such as neurons and ICCs. ${ }^{45-48}$ ICCs play an important part in colonic motility: propulsion is considered to be induced by ICCs in the submucosal plexus, and the ICCs in muscle layers are thought to mediate between the nerves and the smooth muscle cells. ${ }^{49}$ According to a previous human study and the present study, the concentration of ICCs seems to decrease in old age. ${ }^{10}$ In addition, the number of ICCs was found to decline in the colons of humans with slow-transit constipation. ${ }^{50}$ As analyzed with IHC in this study, the area containing KIT-positive ICCs diminished in old age and the rate of decrease was greatest in the myenteric plexus. Therefore, it is suggested that a decrease in KITpositive ICCs via senescence contributes to colonic dysmotility in old age.

Several studies using human or mouse colons have reported changes in the enteric nervous system with age. In humans, cholin- 
ergic neurons are particularly vulnerable to neuronal loss in the myenteric plexus caused by aging. ${ }^{24}$ In mice, myenteric neurons were maintained in aging DC. ${ }^{51}$ In rats, there were no losses of nitrergic neurons stained with nicotinamide adenine dinucleotide phosphate diaphorase (NADPHd) in the stomach, small intestine, or large intestine. ${ }^{25}$ However, the total population of neurons stained with Cuprolinic Blue was reduced in both the aged small intestine and large intestine. ${ }^{25}$ On the other hand, decreased staining intensity for NADPHd-positive neurons and reduced nNOS expression in the myenteric plexus of aged F344BNF1 hybrid rats have been reported. ${ }^{27,28}$ In our previous study on the AC of F344 rats, we showed that the number of $\mathrm{nNOS}$-positive neuronal cells declined with age, as analyzed with the nNOS/PGP 9.5 ratio and the percentage of nNOS-positive neuronal cells per total neuronal cells in myenteric ganglia. ${ }^{14}$ In the present study, in the $\mathrm{AC}$ and DC, neuronal areas as indicated with PGP 9.5-immunoreactive areas increased in adolescence, and decreased to the level of young rats in old age; the loss of myenteric neurons with age was consistent with previous reports in aged mice and rats. ${ }^{51,52}$ In addition, nNOS expression visible on Western blotting decreased according to age. However, there was a slight increase in 2-year old rats compared to 74-week old rats. Although the nNOS-positive areas in the submucosal plexus and myenteric plexus were reduced in old age, as analyzed with the nNOS/PGP 9.5 ratio to exclude the effect of dilution with growth, the decrease of nNOS in the myenteric plexus was not statistically significant. Despite this limitation, age-related loss of $\mathrm{nNOS}$ in the $\mathrm{DC}$ is supported by the decrease in colonic transit, nNOS protein level, NO production and the EFS response.

The significant age-related decrease of NO level in the DC described in this study was consistent with the results of previous studies that showed attenuation of nNOS expression in the colonic myenteric plexus of aged rats (Fig. 6A). ${ }^{27}$ These results suggest that a reduction of nNOS-positive neurons may be associated to colonic aging in the DC. This data also supports the previous results showing that colonic relaxation decreased particularly in the DC, which caused the colon to be feasible to be susceptible to diverticulum, resulting in colonic dysmotility. ${ }^{53}$

The maintenance of $\mathrm{KCl}$ response in old age may reflect conservation of the function of the muscle cell itself. ${ }^{23,54}$ Figure $6 \mathrm{C}$ shows a comparison of contraction before and after treatment with L-NAME in the resting state without EFS. If NO majorly contributes to the contraction in the resting state, spontaneous contraction is expected to increase after blocking NO. Inhibition by LNAME will be altered if the activity of excitatory components such as tachykinin exceeds the inhibitory activity. According to results of this study, the contribution of NO to motility was more prominent in 6-week old rats than in 2-year old rats, in the circulatory muscles of the AC and DC. The contribution of NO in the longitudinal muscle seems to be relatively low.

The functional consequences of declined nNOS expression on colonic transit are incompletely understood. In the case of the colonic pseudo-obstruction in patients with intractable constipation, the reduced motility could be related to nNOS depletion especially in the transition zone close to the splenic flexure. ${ }^{55}$ These findings suggest that the changes in the nNOS-positive neurons may explain the impaired motility due to senescence. In the present study, EFS was conducted at frequencies of 1, 2, 5, 10, and $20 \mathrm{~Hz}$. With EFS at and above $10 \mathrm{~Hz}$, neuropeptides other than acetylcholines are secreted, and their functions are not different from acetylcholines. ${ }^{56}$ Such neuropeptides include excitatory neuropeptides such as neurokinin $\mathrm{A}$ and $\mathrm{B}$, substance $\mathrm{P}$, and inhibitory neuropeptides such as vasoactive intestinal peptides, gastric inhibitory polypeptides, neuropeptide $\mathrm{S}$, and galanin. ${ }^{57}$ The response to EFS in the present result can be interpreted by the balance between the excitatory and inhibitory responses (Fig. 7). The excitatory response was predominant over the inhibitory response in older rats, while the inhibitory response was superior to the excitatory response in younger rats, especially in the distal circular function (Fig. 7D). Contractility was not increased in the older rats after treatment of L-NAME as compared to the point after treatment of apamin, which suggests a reduction of the contribution of $\mathrm{NO}$ to the distal circular function. Thus, the decrease of the contribution of NO to the inhibitory response was observed in the circular direction in the DC (Fig. 6C and 7D), and was consistent with the NO level. On the other hand, the increase of EFS response in the distal longitudinal muscle of older rats may be a compensation for functional damage to the distal circular muscles (Fig. 7B). These results demonstrate that insufficient production of $\mathrm{NO}$ may have influenced dysmotility in the DC of old rats.

In conclusion, the $\mathrm{AC}$ and $\mathrm{DC}$ may differ in their distribution of ICCs and nNOS, and an age-related loss of nitrergic neurons affects more severely the DC than the AC.

\section{Supplementary Material}

Note: To access the supplementary figure mentioned in this article, visit the online version of Journal of Neurogastroenterology and Motility at http://www.jnmjournal.org/, and at https://doi. org/10.5056/jnm17061. 
Financial support: This work was supported by the National Research Foundation of Korea (NRF) grant for the Global Core Research Center (GCRC) funded by the Korea government (MSIP) (No. 2011-0030001). The funders had no role in study design, data collection and analysis, decision to publish, or preparation of the manuscript.

\section{Conflicts of Interest: None.}

Author contributions: Sun Min Lee organized, analyzed, and interpreted the data, and wrote the manuscript; Nayoung Kim designed, and mediated the present study, and supervised the manuscript; Hyun Jin Jo, Ji Hyun Park, Ryoung Hee Nam, Hye Seung Lee, Hyun Jin Kim, and Moon Young Lee performed the experiments and interpreted the data; and Moon Young Lee, Yong Sung Kim, and Dong Ho Lee advised design and supported preparing the manuscript including tables and the figures, and supervised the manuscript.

\section{References}

1. Higgins PD, Johanson JF. Epidemiology of constipation in North America: a systematic review. Am J Gastroenterol 2004;99:750-759.

2. Locke GR 3rd, Pemberton JH, Phillips SF. AGA technical review on constipation. American Gastroenterological Association. Gastroenterology 2000;119:1766-1778.

3. Johanson JF, Kralstein J. Chronic constipation: a survey of the patient perspective. Aliment Pharmacol Ther 2007;25:599-608.

4. Bharucha AE, Wald A, Enck P, Rao S. Functional anorectal disorders. Gastroenterology 2006;130:1510-1518.

5. Lynch AC, Anthony A, Dobbs BR, Frizelle FA. Anorectal physiology following spinal cord injury. Spinal Cord 2000;38:573-580.

6. McCrea GL, Miaskowski C, Stotts NA, Macera L, Varma MG. Pathophysiology of constipation in the older adult. World J Gastroenterol 2008;14:2631-2638.

7. Sanders KM, Ordög T, Ward SM. Physiology and pathophysiology of the interstitial cells of Cajal: from bench to bedside. IV. Genetic and animal models of GI motility disorders caused by loss of interstitial cells of Cajal. Am J Physiol Gastrointest Liver Physiol 2002;282:G747-G756.

8. Sanders KM. A case for interstitial cells of Cajal as pacemakers and mediators of neurotransmission in the gastrointestinal tract. Gastroenterology 1996;111:492-515.

9. Huizinga JD, Thuneberg L, Klüppel M, Malysz J, Mikkelsen HB, Bemstein A. W/kit gene required for interstitial cells of Cajal and for intestinal pacemaker activity. Nature 1995;373:347-349.

10. Gomez-Pinilla PJ, Gibbons SJ, Sarr MG, et al. Changes in interstitial cells of Cajal with age in the human stomach and colon. Neurogastroenterol Motil 2011;23:36-44.

11. Chen H, Redelman D, Ro S, Ward SM, Ordög T, Sanders KM. Se- lective labeling and isolation of functional classes of interstitial cells of Cajal of human and murine small intestine. Am J Physiol Cell Physiol 2007;292:C497-C507.

12. Tong WD, Liu BH, Zhang LY, Xiong RP, Liu P, Zhang SB. Expression of c-kit messenger ribonucleic acid and c-kit protein in sigmoid colon of patients with slow transit constipation. Int J Colorectal Dis 2005;20:363-367.

13. Yamamoto T, Watabe K, Nakahara M, et al. Disturbed gastrointestinal motility and decreased interstitial cells of Cajal in diabetic $\mathrm{db} / \mathrm{db}$ mice. J Gastroenterol Hepatol 2008;23:660-667.

14. Jo HJ, Kim N, Nam RH, et al. Fat deposition in the tunica muscularis and decrease of interstitial cells of Cajal and nNOS-positive neuronal cells in the aged rat colon. Am J Physiol Gastrointest Liver Physiol 2014;306:G659-G669.

15. Ordog T, Hayashi Y, Gibbons SJ. Cellular pathogenesis of diabetic gastroenteropathy. Minerva Gastroenterol Dietol 2009;55:315-343.

16. Wu JJ, Rothman TP, Gershon MD. Development of the interstitial cell of Cajal: origin, kit dependence and neuronal and nonneuronal sources of kit ligand. J Neurosci Res 2000;59:384-401.

17. Phillips RJ, Powley TL. Innervation of the gastrointestinal tract: patterns of aging. Auton Neurosci 2007;136:1-19.

18. Camilleri M, Lee JS, Viramontes B, Bharucha AE, Tangalos EG. Insights into the pathophysiology and mechanisms of constipation, irritable bowel syndrome, and diverticulosis in older people. J Am Geriatr Soc 2000;48:1142-1150

19. Varma JS, Bradnock J, Smith RG, Smith AN. Constipation in the elderly. A physiologic study. Dis Colon Rectum 1988;31:111-115.

20. El-Salhy M, Sandstrom O, Holmlund F. Age-induced changes in the enteric nervous system in the mouse. Mech Ageing Dev 1999;107:93103.

21. Santer RM, Baker DM. Enteric neuron numbers and sizes in Auerbach's plexus in the small and large intestine of adult and aged rats. J Auton Nerv Syst 1988;25:59-67.

22. Gabella $G$. Fall in the number of myenteric neurons in aging guinea pigs. Gastroenterology 1989;96:1487-1493.

23. Tran L, Greenwood-Van Meerveld B. In a non-human primate model, aging disrupts the neural control of intestinal smooth muscle contractility in a region-specific manner. Neurogastroenterol Motil 2014;26:410-418.

24. Bernard CE, Gibbons SJ, Gomez-Pinilla PJ, et al. Effect of age on the enteric nervous system of the human colon. Neurogastroenterol Motil 2009;21:746-e46.

25. Phillips RJ, Kieffer EJ, Powley TL. Aging of the myenteric plexus: neuronal loss is specific to cholinergic neurons. Auton Neurosci 2003;106:6983.

26. Wade PR, Cowen T. Neurodegeneration: a key factor in the ageing gut. Neurogastroenterol Motil 2004;16(suppl 1):19-23.

27. Takahashi T, Qoubaitary A, Owyang C, Wiley JW. Decreased expression of nitric oxide synthase in the colonic myenteric plexus of aged rats. Brain Res 2000;883:15-21.

28. Cowen T, Johnson RJ, Soubeyre V, Santer RM. Restricted diet rescues rat enteric motor neurones from age related cell death. Gut 2000;47:653660 
29. Takahashi T, Owyang C. Regional differences in the nitrergic innervation between the proximal and the distal colon in rats. Gastroenterology 1998;115:1504-1512.

30. Mizuta Y, Takahashi T, Owyang C. Nitrergic regulation of colonic transit in rats. Am J Physiol 1999;277(2 Pt 1):G275-G279.

31. Radenkovic G, Abramovic M. Differentiation of interstitial cells of Cajal in the human distal colon. Cells Tissues Organs 2012;196:463-469.

32. Kwak JM, Babygirija R, Gribovskaja-Rupp I, et al. Regional difference in colonic motility response to electrical field stimulation in guinea pig. J Neurogastroenterol Motil 2013;19:192-203.

33. Albertí E, Mikkelsen HB, Larsen JO, Jiménez M. Motility patterns and distribution of interstitial cells of Cajal and nitrergic neurons in the proximal, mid- and distal-colon of the rat. Neurogastroenterol Motil 2005;17:133-147.

34. Ward SM, Gershon MD, Keef K, Bayguinov YR, Nelson C, Sanders KM. Interstitial cells of Cajal and electrical activity in ganglionic and aganglionic colons of mice. Am J Physiol Gastrointest Liver Physiol 2002;283:G445-G456.

35. Hagger R, Gharaie S, Finlayson C, Kumar D. Regional and transmural density of interstitial cells of Cajal in human colon and rectum. Am J Physiol 1998;275(6 Pt 1):G1309-G1316.

36. Horisawa M, Watanabe Y, Torihashi S. Distribution of c-Kit immunopositive cells in normal human colon and in Hirschsprung's disease. J Pediatr Surg 1998;33:1209-1214.

37. Torihashi S, Horisawa M, Watanabe Y. c-Kit immunoreactive interstitial cells in the human gastrointestinal tract. J Auton Nerv Syst 1999;75:3850.

38. Million M, Maillot C, Saunders P, Rivier J, Vale W, Taché Y. Human urocortin II, a new CRF-related peptide, displays selective CRF2mediated action on gastric transit in rats. Am J Physiol Gastrointest Liver Physiol 2002;282:G34-G40.

39. Park JH, Kwon JG, Kim SJ, et al. Alterations of colonic contractility in an interleukin-10 knockout mouse model of inflammatory bowel disease. J Neurogastroenterol Motil 2015;21:51-61.

40. Nakahara M, Isozaki K, Hirota S, et al. Deficiency of KIT-positive cells in the colon of patients with diabetes mellitus. J Gastroenterol Hepatol 2002;17:666-670.

41. Grider JR, Murthy KS, Jin JG, Makhlouf GM. Stimulation of nitric oxide from muscle cells by VIP: prejunctional enhancement of VIP release. Am J Physiol 1992;262(4 Pt 1):G774-G778.

42. Grider JR. Interplay of VIP and nitric oxide in regulation of the descending relaxation phase of peristalsis. Am J Physiol 1993;264(2 Pt 1):G334G340.

43. Broccardo M, Improta G, Tabacco A. Central tachykinin NK3 receptors in the inhibitory action on the rat colonic propulsion of a new tachykinin, PG-KII. Eur J Pharmacol 1999;376:67-71.

44. Raffa RB, Mathiasen JR, Jacoby HI. Colonic bead expulsion time in normal and mu-opioid receptor deficient (CXBK) mice following central (ICV) administration of mu- and delta-opioid agonists. Life Sci 1987;41:2229-2234.

45. Hoyle CH, Saffrey MJ. Effects of aging on cholinergic neuromuscular transmission in isolated small intestine of ad libitum fed and caloricallyrestricted rats. Neurogastroenterol Motil 2012;24:586-592.

46. Saffrey MJ. Aging of the mammalian gastrointestinal tract: a complex organ system. Age (Dordr) 2014;36:9603.

47. Bashashati M, McCallum RW. Is interstitial cells of Cajal-opathy present in gastroparesis? J Neurogastroenterol Motil 2015;21:486-493.

48. Singh R, Ghoshal UC, Misra A, Mittal B. Achalasia is associated with eNOS4a4a, iNOS22GA, and nNOS29TT genotypes: a case-control study. J Neurogastroenterol Motil 2015;21:380-389.

49. Vanderwinden JM, Rumessen JJ, Bernex F, Schiffmann SN, Panthier JJ. Distribution and ultrastructure of interstitial cells of Cajal in the mouse colon, using antibodies to Kit and Kit W-lacZ mice. Cell Tissue Res 2000;302:155-170.

50. Lyford GL, He CL, Soffer E, et al. Pan-colonic decrease in interstitial cells of Cajal in patients with slow transit constipation. Gut 2002;51:496501.

51. Gamage PP, Ranson RN, Patel BA, Yeoman MS, Saffrey MJ. Myenteric neuron numbers are maintained in aging mouse distal colon. Neurogastroenterol Motil 2013;25:e495-e505.

52. Phillips RJ, Pairitz JC, Powley TL. Age-related neuronal loss in the submucosal plexus of the colon of Fischer 344 rats. Neurobiol Aging 2007;28:1124-1137.

53. Park KS. [Aging and digestive diseases: at the view of the functional change of gastrointestinal tract.] Korean J Gastroenterol 2011;58:3-8. [Korean]

54. Sung TS, La JH, Kang TM, Kim TW, Yang IS. Visceral hypersensitivity and altered colonic motility in type 2 diabetic rat. J Neurogastroenterol Motil 2015;21:581-588.

55. Do YS, Myung SJ, Kwak SY, et al. Molecular and cellular characteristics of the colonic pseudo-obstruction in patients with intractable constipation. J Neurogastroenterol Motil 2015;21:560-570.

56. Cai Y, Tang H, Jiang F, Dong Z. Slow wave activity and modulations in mouse jejunum myenteric plexus in situ. J Neurogastroenterol Motil 2017;23:117-123.

57. Koh SD, Rhee PL. Ionic conductance(s) in response to post-junctional potentials. J Neurogastroenterol Motil 2013;19:426-432. 